PROCEEDINGS OF THE

AMERICAN MATHEMATICAL SOCIETY

Volume 126, Number 10, October 1998, Pages 2945-2948

S 0002-9939(98)04419-0

\title{
ON AN EXAMPLE CONCERNING THE KOBAYASHI PSEUDODISTANCE
}

\author{
WŁODZIMIERZ ZWONEK
}

(Communicated by Steven R. Bell)

\begin{abstract}
In this short note we calculate the Kobayashi pseudodistance for elementary Reinhardt domains in $\mathbb{C}^{2}$. They deliver us a number of examples giving a negative answer to a problem posed by S. Kobayashi.
\end{abstract}

For a domain $D \subset \mathbb{C}^{n}, w, z \in D$ define (see $[\mathrm{K}]$ and $[\mathrm{L}]$ )

$\tilde{k}_{D}(w, z):=\inf \left\{p\left(\lambda_{1}, \lambda_{2}\right):\right.$ there is $\varphi \in \mathcal{O}(E, D)$ such that $\left.\varphi\left(\lambda_{1}\right)=w, \varphi\left(\lambda_{2}\right)=z\right\}$, where $p$ is the Poincaré distance on the unit disk $E$ in $\mathbb{C}$. Let us also define $k_{D}$ as the largest pseudodistance smaller or equal than $\tilde{k}_{D}$. We call $\tilde{k}_{D}$ the Lempert function and $k_{D}$ the Kobayashi pseudodistance of the domain $D$.

It is well known (see $[\mathrm{K}]$ and $[\mathrm{JP}]$ ) that if $F: D \rightarrow G$ is a holomorphic covering $\left(D\right.$ and $G$ are domains in $\left.\mathbb{C}^{n}\right), F(\tilde{w})=w$, then

$$
\tilde{k}_{G}(w, z)=\inf \left\{\tilde{k}_{D}(\tilde{w}, \tilde{z})\right\}, k_{G}(w, z)=\inf \left\{k_{D}(\tilde{w}, \tilde{z})\right\}, w, z \in G,
$$

where the infimum, in both cases, is taken over all $\tilde{z} \in D$ such that $F(\tilde{z})=z$. The problem whether the infimum is always attained was posed in [K], page 48 (in case of the Kobayashi pseudodistance). The analoguous problem concerning the Lempert function was posed in [JP].

Fix $\alpha=\left(\alpha_{1}, \ldots, \alpha_{n}\right) \in\left(\mathbb{R}_{*}\right)^{n}$. Let us denote

$$
D_{\alpha}:=\left\{z \in \mathbb{C}^{n}:\left|z_{1}\right|^{\alpha_{1}} \cdot \ldots \cdot\left|z_{n}\right|^{\alpha_{n}}<1\right\} .
$$

We say that a domain $D_{\alpha}$ (respectively, $\alpha$ ) is of rational type if there are $t \in \mathbb{R}_{+}$ and $\beta=\left(\beta_{1}, \ldots, \beta_{n}\right) \in \mathbb{Z}^{n}$ such that $\alpha=t \beta$. $D_{\alpha}$ (respectively, $\alpha$ ) is of irrational type if it is not of rational type.

In case when $\alpha$ is of rational type, then we always assume that $\alpha \in \mathbb{Z}^{n}$ and all $\alpha_{j}$ 's are relatively prime integers (obtained by multiplying $\alpha$ with some positive number). In that case we denote also $z^{\alpha}=z_{1}^{\alpha_{1}} \cdot \ldots \cdot z_{n}^{\alpha_{n}}$ for all $z \in \mathbb{C}^{n}$ for which it makes sense (in particular if $\alpha_{j}<0$, then $z_{j} \neq 0$ ).

In the paper we restrict our attention only to the two-dimensional case.

Below using the property (1) we shall give effective formulas for both the Lempert function and the Kobayashi pseudodistance, in the case when at least one $\alpha_{j}$ is negative (they turn out to be equal on the considered domains). Additionally, as a simple conclusion from the formulas obtained, we get that the domains $D_{\alpha} \subset \mathbb{C}^{2}$

Received by the editors February 28, 1997.

1991 Mathematics Subject Classification. Primary 32H15.

The paper was partially supported by the KBN grant No 2 PO3A 06008 .

(C)1998 American Mathematical Society 
(with at least one $\alpha_{j}$ negative) of irrational type deliver us examples answering (in a negative way) the above mentioned problem of S. Kobayashi. It seems to be the first known example of that kind.

Since the formulas for the functions involved in case when $\alpha_{j}$ 's are all positive were obtained in [PZ], the results below may be treated as the continuation of that paper.

It is well known that $\tilde{k}_{E}=p$ and that the Kobayashi pseudodistance and the Lempert function coincide on the class of domains in $\mathbb{C}$. We shall make use also of the explicit formula for the function $\tilde{k}_{E_{*}}$ (see e.g. [JP]), where $E_{*}:=E \backslash\{0\}$ :

$$
\begin{aligned}
& \tanh \left(\tilde{k}_{E_{*}}(a, \lambda)\right)= \\
& \left(\frac{\theta^{2}+(\log |\lambda|-\log a)^{2}}{\theta^{2}+(\log |\lambda|+\log a)^{2}}\right)^{1 / 2}, a \in(0,1), \lambda \in E_{*}, \theta:=\operatorname{Arg}(\lambda) \in \arg (\lambda) \cap(-\pi, \pi] .
\end{aligned}
$$

Theorem 1. 1. In case $\alpha_{1}>0$ and $\alpha_{2}<0$ we have if $D_{\alpha}$ is of rational type, then

$$
\tilde{k}_{D_{\alpha}}(w, z)=k_{D_{\alpha}}(w, z)=\min \left\{p\left(\lambda_{1}, \lambda_{2}\right): \lambda_{1}^{\alpha_{1}}=w^{\alpha}, \lambda_{2}^{\alpha_{1}}=z^{\alpha}, \lambda_{1}, \lambda_{2} \in E\right\} ;
$$

if $D_{\alpha}$ is of irrational type, then

$$
\tilde{k}_{D_{\alpha}}(w, z)=k_{D_{\alpha}}(w, z)=p\left(\left(\left|w_{1}\right|^{\alpha_{1}}\left|w_{2}\right|^{\alpha_{2}}\right)^{1 / \alpha_{1}},\left(\left|z_{1}\right|^{\alpha_{1}}\left|z_{2}\right|^{\alpha_{2}}\right)^{1 / \alpha_{1}}\right), w, z \in D_{\alpha} .
$$

2. In case $\alpha_{1}, \alpha_{2}<0$ we have

if $D_{\alpha}$ is of rational type, then

$$
\tilde{k}_{D_{\alpha}}(w, z)=k_{D_{\alpha}}(w, z)=\tilde{k}_{E_{*}}\left(w^{\alpha}, z^{\alpha}\right) ;
$$

if $\alpha$ is of irrational type, then

$$
\tilde{k}_{D_{\alpha}}(w, z)=k_{D_{\alpha}}(w, z)=\tilde{k}_{E_{*}}\left(\left|w_{1}\right|^{\alpha_{1}}\left|w_{2}\right|^{\alpha_{2}},\left|z_{1}\right|^{\alpha_{1}}\left|z_{2}\right|^{\alpha_{2}}\right), w, z \in D_{\alpha}
$$

Proof. Let us consider the case $\alpha_{1}>0, \alpha_{2}<0$. Define the mapping

$$
\Phi: E \times \mathbb{C} \ni(\lambda, \mu) \rightarrow\left(\lambda \exp \left(\alpha_{2} \mu\right), \exp \left(-\alpha_{1} \mu\right)\right) \in D_{\alpha}
$$

The mapping $\Phi$ is locally biholomorphic; moreover, one may easily verify that it is a holomorphic covering.

The condition $\Phi\left(\lambda_{1}, \mu_{1}\right)=w$ and $\Phi\left(\lambda_{2}, \mu_{2}\right)=z$ is equivalent to the following:

$$
\begin{gathered}
\mu_{1}=-\frac{1}{\alpha_{1}} \log \left|w_{2}\right|-\frac{\left(2 k_{1} \pi+\operatorname{Arg}\left(w_{2}\right)\right) i}{\alpha_{1}} \\
\lambda_{1}=w_{1}\left|w_{2}\right|^{\alpha_{2} / \alpha_{1}} \exp \left(\left(2 k_{1} \pi+\operatorname{Arg}\left(w_{2}\right)\right) i \frac{\alpha_{2}}{\alpha_{1}}\right) \\
\mu_{2}=-\frac{1}{\alpha_{1}} \log \left|z_{2}\right|-\frac{\left(2 k_{2} \pi+\operatorname{Arg}\left(z_{2}\right)\right) i}{\alpha_{1}} \\
\lambda_{2}=z_{1}\left|z_{2}\right|^{\alpha_{2} / \alpha_{1}} \exp \left(\left(2 k_{2} \pi+\operatorname{Arg}\left(z_{2}\right)\right) i \frac{\alpha_{2}}{\alpha_{1}}\right), \text { for some } k_{1}, k_{2} \in \mathbb{Z}
\end{gathered}
$$

Fix $k_{1}=0$ (consequently, we fix $\mu_{1}$ and $\lambda_{1}$ ). Then due to the product property of the Lempert function (see $[\mathrm{JP}]$ ), the relations above and (1) we get

$$
\begin{aligned}
& \tilde{k}_{D_{\alpha}}(w, z)=\inf \left\{\tilde{k}_{E \times \mathbb{C}}\left(\left(\lambda_{1}, \mu_{1}\right),\left(\lambda_{2}, \mu_{2}\right)\right): \Phi\left(\lambda_{2}, \mu_{2}\right)=z\right\}= \\
& \quad \inf \left\{\tilde{k}_{E}\left(\lambda_{1}, \lambda_{2}\right): \lambda_{2}=z_{1}\left|z_{2}\right|^{\alpha_{2} / \alpha_{1}} \exp \left(\left(2 k_{2} \pi+\operatorname{Arg}\left(z_{2}\right)\right) i \frac{\alpha_{2}}{\alpha_{1}}\right), k_{2} \in \mathbb{Z}\right\} .
\end{aligned}
$$


Note that in the rational case the last infimum is taken over the finite set, or more precisely, the $\lambda_{2}$ 's run over all possible $\alpha_{1}$-st roots of $z^{\alpha}$. This completes the proof in that case. If $\alpha$ is of irrational type, then the set

$$
\left\{z_{1}\left|z_{2}\right|^{\alpha_{2} / \alpha_{1}} \exp \left(\left(2 k_{2} \pi+\operatorname{Arg}\left(z_{2}\right)\right) i \frac{\alpha_{2}}{\alpha_{1}}\right), k_{2} \in \mathbb{Z}\right\}
$$

is, in view of the Kronecker theorem (see e.g. [HW]), dense in $\left(\left|z_{1}\right|^{\alpha_{1}}\left|z_{2}\right|^{\alpha_{2}}\right)^{1 / \alpha_{1}} \partial E$. This together with the invariance of $\tilde{k}_{E}(=p)$ with respect to rotations finishes the proof for the Lempert function. Let us observe that the Lempert function in both cases satisfies the triangle inequality, therefore the Kobayashi pseudodistance coincides with the Lempert function.

The proof of the case $\alpha_{1}, \alpha_{2}<0$ goes along the same lines as the first one. Namely, we define the mapping

$$
\Psi: E_{*} \times \mathbb{C} \ni(\lambda, \mu) \rightarrow\left(\frac{\exp \left(\alpha_{2} \mu\right)}{\lambda}, \exp \left(-\alpha_{1} \mu\right)\right) \in D_{\alpha},
$$

which is again a holomorphic covering.

The condition $\Psi\left(\lambda_{1}, \mu_{1}\right)=w$ and $\Psi\left(\lambda_{2}, \mu_{2}\right)=z$ is equivalent to the following

$$
\begin{gathered}
\mu_{1}=-\frac{1}{\alpha_{1}} \log \left|w_{2}\right|-\frac{\left(2 k_{1} \pi+\operatorname{Arg}\left(w_{2}\right)\right) i}{\alpha_{1}}, \\
1 / \lambda_{1}=w_{1}\left|w_{2}\right|^{\alpha_{2} / \alpha_{1}} \exp \left(\left(2 k_{1} \pi+\operatorname{Arg}\left(w_{2}\right)\right) \frac{i \alpha_{2}}{\alpha_{1}}\right), \\
\mu_{2}=-\frac{1}{\alpha_{1}} \log \left|z_{2}\right|-\frac{\left(2 k_{2} \pi+\operatorname{Arg}\left(z_{2}\right) i\right)}{\alpha_{1}}, \\
1 / \lambda_{2}=z_{1}\left|z_{2}\right|^{\alpha_{2} / \alpha_{1}} \exp \left(\left(2 k_{2} \pi+\operatorname{Arg}\left(z_{2}\right)\right) \frac{i \alpha_{2}}{\alpha_{1}}\right), k_{1}, k_{2} \in \mathbb{Z} .
\end{gathered}
$$

Fix $k_{1}=0$ (so $\lambda_{1}$ and $\mu_{1}$, too). Then as in the first case we get

$$
\begin{aligned}
& \tilde{k}_{D_{\alpha}}(w, z)=\inf \left\{\tilde{k}_{E_{*} \times \mathbb{C}}\left(\left(\lambda_{1}, \mu_{1}\right),\left(\lambda_{2}, \mu_{2}\right)\right): \Psi\left(\lambda_{2}, \mu_{2}\right)=z\right\}= \\
& \quad \inf \left\{\tilde{k}_{E_{*}}\left(\lambda_{1}, \lambda_{2}\right): 1 / \lambda_{2}=z_{1}\left|z_{2}\right|^{\alpha_{2} / \alpha_{1}} \exp \left(\left(2 k_{2} \pi+\operatorname{Arg}\left(z_{2}\right)\right) \frac{i \alpha_{2}}{\alpha_{1}}\right), k_{2} \in \mathbb{Z}\right\} .
\end{aligned}
$$

Similarly as in the first case we get the formula

$$
\tilde{k}_{D_{\alpha}}(w, z)=\min \left\{\tilde{k}_{E_{*}}\left(\lambda_{1}, \lambda_{2}\right): \lambda_{1}^{-\alpha_{1}}=w^{\alpha}, \lambda_{2}^{-\alpha_{1}}=z^{\alpha}\right\}
$$

To get the desired formula, i.e. to get rid of the minimum and the roots, it is sufficient to make use of the formula for $\tilde{k}_{E_{*}}$ (it needs some elementary calculations to be omitted here). This completes the proof in that case.

If $\alpha$ is of the irrational type, then the set

$$
\left\{z_{1}\left|z_{2}\right|^{\alpha_{2} / \alpha_{1}} \exp \left(\left(2 k_{2} \pi+\operatorname{Arg}\left(z_{2}\right)\right) i \frac{\alpha_{2}}{\alpha_{1}}\right), k_{2} \in \mathbb{Z}\right\}
$$

is, in view of the Kronecker theorem, dense in $\left(\left|z_{1}\right|^{\alpha_{1}}\left|z_{2}\right|^{\alpha_{2}}\right)^{1 / \alpha_{1}} \partial E$. This together with the invariance of $\tilde{k}_{E_{*}}$ with respect to rotations gives us the formula

$$
\tilde{k}_{D_{\alpha}}(w, z)=\tilde{k}_{E_{*}}\left(\left(\left|w_{1}\right|^{\alpha_{1}}\left|w_{2}\right|^{\alpha_{2}}\right)^{-1 / \alpha_{1}},\left(\left|z_{1}\right|^{\alpha_{1}}\left|z_{2}\right|^{\alpha_{2}}\right)^{-1 / \alpha_{1}}\right) .
$$

And now, the effective formula for the function $\tilde{k}_{E_{*}}$ allows us to get rid of the powers in the formula, which completes the proof (the idea of getting rid of the powers in the formula in the second case is due to Prof. P. Pflug). 
Let us observe that the Lempert function in both cases satisfies the triangle inequality, therefore the Kobayashi pseudodistance coincides with the Lempert function.

Remark 2. Note that if $\alpha$ is of irrational type, then from the proof of Theorem 1 (especially of the form of $\Phi$ and $\Psi$ ) we get that the infimum (as described in (1)) need not be attained. To visualize that fix for example $\alpha_{1}=1, \alpha_{2}<-1, \alpha_{2} \notin \mathbb{Q}$, $w=(s, s)$ and $t=(s,-s)$, where $s>1$. Then $w, z \in D_{\alpha}, \operatorname{Arg}(s)=0$ whereas $\operatorname{Arg}(-s)=\pi$ and

$$
\tilde{k}_{D_{\alpha}}(w, z)=k_{D_{\alpha}}(w, z)=0=\inf \left\{\tilde{k}_{E}\left(s^{1+\alpha_{2}}, s^{1+\alpha_{2}} \exp \left(\left(2 k_{2}+1\right) i \pi \alpha_{2}\right)\right), k_{2} \in \mathbb{Z}\right\}
$$

The infimum is as in the proof of Theorem 1; it represents the infimum from the formula (1) for the holomorphic covering $\Phi$. It is evident that the infimum is not attained by any $k_{2} \in \mathbb{Z}$. The similar procedure goes for the case $\alpha_{1}, \alpha_{2}<0$ and $\alpha$ being of irrational type.

Remark 3. Let us notice here that it is possible to calculate the formulas for the Lempert function and the Kobayashi pseudodistance for all elementary Reinhardt domains (in arbitrary dimension). Nevertheless, since the idea is exactly the same as above, the obtained formulas deliver us no new phenomena, and the proof is more complicated, we skip it here.

\section{REFERENCES}

[HW] G. H. Hardy \& E. M. Wright, An Introduction to the Theory of Numbers, Oxford Science Publ., 1978. MR 81i: 10002

[JP] M. Jarnicki \& P. Pflug, Invariant Distances and Metrics in Complex Analysis, Walter de Gruyter, 1993. MR 94k:32039

[K] S. Kobayashi, Hyperbolic Manifolds and Holomorphic Mappings, Pure and Appl. Math. 2, M. Dekker, 1970. MR 43:3503

[L] L. Lempert, La métrique de Kobayashi et la représentation des domaines sur la boulle, Bull. Soc. Math. France 109 (1981), 427-479. MR 84d:32036

[PZ] P. Pflug \& W. Zwonek, Effective formulas for invariant functions - case of elementary Reinhardt domains, (preprint).

Instytut Matematyki, Uniwersytet Jagielloński, Reymonta 4, 30-059 Kraków, Poland

E-mail address: zwonek@im.uj.edu.pl

Current address: Carl von Ossietzky Universität Oldenburg, Fachbereich 6-Mathematik, Postfach 2503, 26111 Oldenburg, Germany

E-mail address: zwonek@mathematik.uni-oldenburg.de 